\title{
Schienentherapie in der gesetzlichen Krankenversicherung
}

\begin{abstract}
Als ein Zeichen der Zeit nehmen die "Zerknirrschtheit" der Bevölkerung und damit die Notwendigkeit von Therapien der Folgeerkrankungen zu. Die immer größer werdende Informationsflut sowie die "Vereinsamung" der Menschen in der mobiler werdenden Gesellschaft nehmen mit allen psychischen Folgeerkrankungen einen immer größeren Stellenwert auch als Kostenfaktor für die Krankenkassen ein. Auch die Therapie mit Schienen ist somit zu einer nicht zu unterschätzenden Größe angestiegen.
\end{abstract}

Um es vorweg zu nehmen: Es gibt in jedem Bundesland besondere spezielle Regulierungen zum Budget und Honorarverteilungsmaßstab (HVM). Diese Regelungen sollten natürlich zusätzlich beachtet werden. Denn ungeachtet der erbrachten Leistung kann es sein, dass nur ein Teil der abgerechneten Summe oder nur eine gewisse Anzahl von Behandlungen zur Auszahlung kommt. Dies sollte der Zahnarzt immer im Hinterkopf haben.

Ein Behandlungsplan für Kiefergelenkserkrankungen und Kieferbruch muss vor Erbringung der Leistungen zur Genehmigung bei der betreffenden Krankenkasse eingereicht werden. Hiervon gibt es in einzelnen KZV-Bereichen Ausnahmen wegen Sonderverträgen mit einzelnen Krankenkassen. Es ist möglich, in der Abrechnungsmappe oder dem Internetauftritt der zuständigen KZV abzuklären, welche Sondervereinbarungen es gibt. Ebenfalls ohne vorherige Genehmigung kann eine Behandlung zur Beseitigung akuter Schmerzen durchgeführt werden. Die Dokumentation spielt hier eine wichtige Rolle, damit der Nachweis der Schmerztherapie geführt werden kann. Die schmerztherapeutische Schiene ohne adjustierte Oberfläche kann mit der BEMA-Nummer K2 abgerechnet werden. Auch die einleitende Maßnahme zur Muskelrelaxation kann abgerechnet werden, aber hier mit vorheriger Genehmigung mit der Nummer K2. Ebenso ist die Strahlenschutzschiene (Weichgewebsretraktor) als „Sonderfall“ ausnahmslos genehmigungspflichtig als K2 abrechenbar. Diese Formen der Schienentherapie können nicht in zeitlichem Zusammenhang mit den BEMANummern K1-K3 im selben Kiefer abgerechnet werden.

\section{Viele Formen der Versorgung}

Die BEMA-Leistung K1 „Eingliedern eines Aufbissbehelfes mit adjustierter Oberfläche" dient zur Unterbrechung der Okklusionskontakte oder als Aufbissschiene bei PA-Behandlungen oder als Bissführungsplatte bei der Versorgung mit Zahnersatz. Es geht hierbei nur um individuelle Schienen.

Die entsprechenden Leistungen im Labor werden mit den erbrachten Leistungen nach K1-K3 auf dem entsprechenden Abrechnungsformular abgerechnet. Je nach Bundesland werden Portokosten, Abformmaterial und Versandgänge übernommen.

Die BEMA-Nummer K3 dient der Abrechnung der „Umarbeitung einer vorhandenen Prothese zum Aufbissbehelf zur Unterbrechung der Okklusionskontakte mit adjustierter Oberfläche" und kann nur bei vorhandener herausnehmbarer prothetischer Versorgung erfolgen.

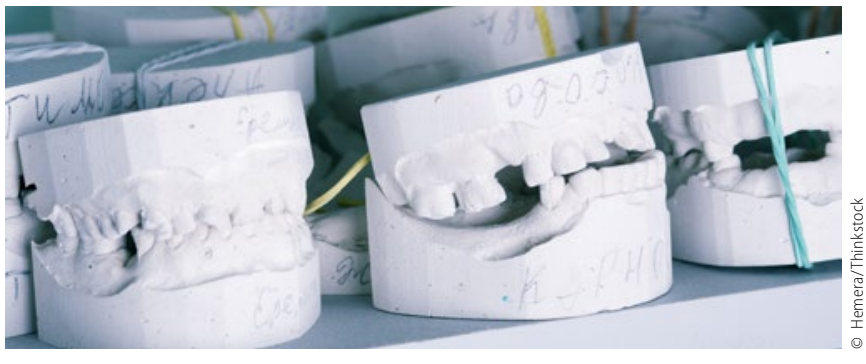

Die Schienentherapie ist ein weites Feld und umfasst viele Formen der Versorgung. Deshalb kann die Berechnung der erbrachten Leistung große Unterschiede aufweisen. Falls eine Übertragung der Oberkieferposition zur Einstellung der Modelle in den Artikulator nötig sein sollte, müsste diese über die GOZ mit dem Patienten sowohl vereinbart als auch berechnet werden. Dazu müssten vor Behandlungsbeginn eine schriftliche Vereinbarung nach $₫ 4$ Abs. 5 BMV - Z bzw. $\$ 7$ Abs.7 EKVZ, eine schriftliche Vereinbarung nach $\$ 2$ Abs. $3 \mathrm{GOZ}$ sowie ein Kostenplan nach GOZ getroffen und ausgehändigt werden. Weitere Leistungen nach GOZ aus dem Bereich der FAL/FTL unterliegen den gleichen Vorgaben, sofern sie im Rahmen der Behandlung nötig werden sollten.

Sportlerschutz wie auch bimaxilläre Therapeutika und Schnarcherschienen (siehe Artikel Dr. Dr. Raff, Seite 54) sind nicht Gegenstand der vertragszahnärztlichen Versorgung und müssen privat vereinbart und berechnet werden.

Eine „Semipermanente Schienung unter Anwendung der Ätztechnik" ist je Interdentalraum mit der BEMA-Nummer K4 abrechenbar. Wird mit Fasern oder Draht verstärkt, ist der Leistungsinhalt der GOÄ-Nummer 2697 abgedeckt und diese Leistung zu berechnen. Auch ein Retainer ist damit nicht gemeint und somit nicht abrechenbar.

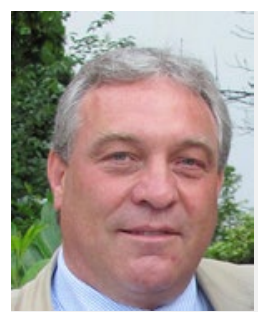

Dr. Christian Öttl

Mitglied im FVDZ-Bundesvorstand Ressort Honorierung 